\title{
MODELO DE ECUACIONES ESTRUCTURALES PARA PREDECIR LA CALIDAD DE NOTIFICACIÓN DE REACCIONES ADVERSAS A MEDICAMENTOS EN LA REGIÓN DE SALUD TACNA
}

\author{
STRUCTURAL EQUATION MODEL \\ TO PREDICT THE QUALITY OF \\ NOTIFICATION OF ADVERSE \\ DRUG REACTIONS IN THEHEALTH \\ REGION OF TACNA
}

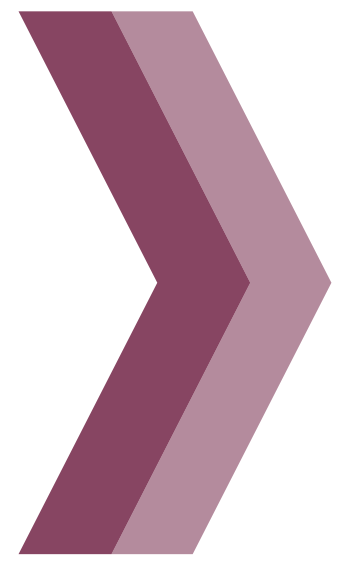

\section{RESUMEN}

OBJETIVO: Diseñar un modelo estructural multifactorial que explique la calidad de la notificación espontánea de reacciones adversas a medicamentos (RAMs) a partir de algunos factores asociados al reporte en establecimientos de la región de salud Tacna. MATERIALES Y MÉTODOS: Estudio predictivo y transversal, mediante encuesta en 30 profesionales de la salud en el 2013. Se usó cuatro instrumentos de recolección: cuestionario para las características demográficas-laborales, test de conocimientos, escala de Likert para medir la actitud hacia el reporte de RAMs y una ficha de cotejo con la que se identificó las relaciones entre las variables de interés. Se probaron tres modelos de ecuaciones estructurales.RESULTADOS: EI modelo estructural de ajuste más satisfactorio relacionó las características demográficas-laborales con la calidad de la notificación espontanea, evidenciando la existencia de mayor incidencia de las variables causales que de las variables intermedias en la notificación espontánea como el peso del parámetro de la experiencia profesional que incide con un valor de $=0,5$, seguida del conocimiento de RAMS con $=0,23$. CONCLUSIÓN: Se validó el modelo por medio de ecuaciones estructurales sobre la base teórica de la experiencia y reespecificado estadísticamente, lo cual brinda información necesaria para desarrollar políticas orientadas a niveles de acción complementaria.

Palabras clave: Ecuaciones estructurales, metodología causal, modelo, notificación espontánea, reacciones adversas.

\section{Juan José E. Changllio Roas}

1. Químico Farmacéutico, Doctor en Epidemiologia, Especialista en Toxicología y control alimentario. Docente de la Facultad de Ciencias de la salud de laUNJBG.

\section{ABSTRACT}

OBJECTIVE: to design a multifactorial structural model that explains the quality of spontaneous notification of adverse drug reactions (ADRs) based on some factors associated with reporting in establishments in the health region of Tacna. MATERIALS AND METHODS: Predictive and cross-sectional study, through a survey of 30 health professionals in 2013. Four collection instruments were used: questionnaire for demographic-labor characteristics, knowledge test, Likert scale to measure attitude towards the report of RAMs and a check sheet with which the relationships among the variables of interest were identified. Three models of structural equations were tested. RESULTS: The most satisfactory structural adjustment model linked the demographic-labor characteristics with the quality of the spontaneous notification, evidencing the existence of a greater incidence of the causal variables than of the intermediate variables in the spontaneous notification as the weight of the professional experience parameter that affects with a value $=0,5$, followed by knowledge of RAMS with $=0,23$. CONCLUSION: the model was validated by means of structural equations on the theoretical basis of the experience and statistically respecified, which provides necessary information to develop policies oriented to levels of complementary action.

Key words: Structural equation, causal methodology, model, spontaneous reporting, adverse reactions. 


\section{INTRODUCCIÓN}

En los países desarrollados, las Reacciones Adversas a los Medicamentos (RAMs) constituyen parte de las primeras razones de morbimortalidad. Se ha estimado que entre un 10 y un $17 \%$ de visitas a servicios de urgencias hospitalarias son producidas por RAMs (7).

Esta situación en América Latina es más preocupante, debido al probable incremento de uso inadecuado de medicamentos en pacientes hospitalizados y ambulatorios. Por tanto, se aprecia una elevada y constante tasa de crecimiento de resistencia bacteriana a antibióticos en infecciones adquiridas tanto en la comunidad como en el hospital.

En nuestro país, se dispone de escasa información del número real de RAMs, habiéndose reportado un mayor número de notificaciones RAMs en el servicio de tomografía $(47,22 \%)$ en el Hospital Nacional "Guillermo Almenara Irigoyen" de Lima durante el periodo de enero a julio del 2003, seguido de los servicios que comprende a las diferentes especialidades $(28,89 \%)$. Los medios de contraste iodados (46,30 \%) y los antibacterianos (22,04\%) fueron considerados como agentes responsables.

En el 2010, el Instituto Nacional de Enfermedades Neoplásicas (INEN) comunicó que una niña de cuatro años falleció a causa de los efectos de RAMs, tras recibir un medicamento oncológico como parte de su tratamiento. Otros tres niños que se atendieron en dicho nosocomio también presentaron RAMs. Esta situación evidencia la relevancia del problema y la necesidad de indagar factores o variables que se vinculan con el deficiente reporte de RAMs o su ausencia.

Es indudable que todas sospecha de RAMs deberia ser reportada, ya que las consecuencias tienen carácter mórbido. En algunos casos, se requiere hospitalización; en otros, intervenciones médicas o quirúrgicas, y en otros, el desenlace es fatal. Sin embargo, se sabe que muchas RAMs no llegan a ser identificadas como tales; adicionalmente, una gran parte de ellas especialmente las de menor gravedad- tienden a no ser reportadas. Consecuentemente, el impacto que tienen las RAMs está subestimado, razón por la cual asumimos que los factores asociados a la notificación de RAMs serían: la exhaustividad de la información, la integralidad de los datos y la oportunidad de entrega de la notificación a los organismos correspondientes. También son asociados otros factores, tales como el conocimiento, las actitudes y las prácticas de los profesionales de la salud frente a la farmacovigilancia, la notificación espontánea de RAMs, así como la disponibilidad de tiempo del profesional. Esto porque adicionalmente a las tareas asistenciales, el profesional desarrolla labores administrativas. Por otro lado, también se observa que el usuario y/o paciente muchas veces no advierte la presencia de una RAM o desconoce el procedimiento de reporte ante un establecimiento de salud público o privado, lo que menoscaba la información oficial.

\section{MATERIALESY MÉTODOS}

Estudio de corte transversal en 30 profesionales de la salud que reportaron reacciones adversas durante el 2013. Para recopilar los datos, se utilizó un cuestionario para medir las características socio-demográficas y laborales: edad, sexo, grupo profesional, experiencia, y centro de trabajo. También se aplicó un test de conocimiento con 17 ítems con el fin de recopilar datos para las actitudes, se utilizó un formato de Likert conformado por 20 ítems con 5 niveles de respuesta y una ficha de evaluación del formato de reporte de RAMS. Esta última estaba conformada por veintiocho ítems que miden la exhaustividad, la pertinencia, la legibilidad, la integralidad y la oportunidad de la notificación espontánea de RAMs.

Para la estadística descriptiva, se usó el paquete estadístico SPSS. Además, para la estimación y ajuste de los modelos estructurales, se utilizó el programa LISREL.

\section{RESULTADOS}

Se formuló un modelo a partir de la revisión de la literatura, identificando las variables siendo expresadas en ecuaciones estructurales, tal como lo demuestra la figura 1 . de conocimiento y sus características demográficas. 


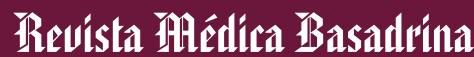

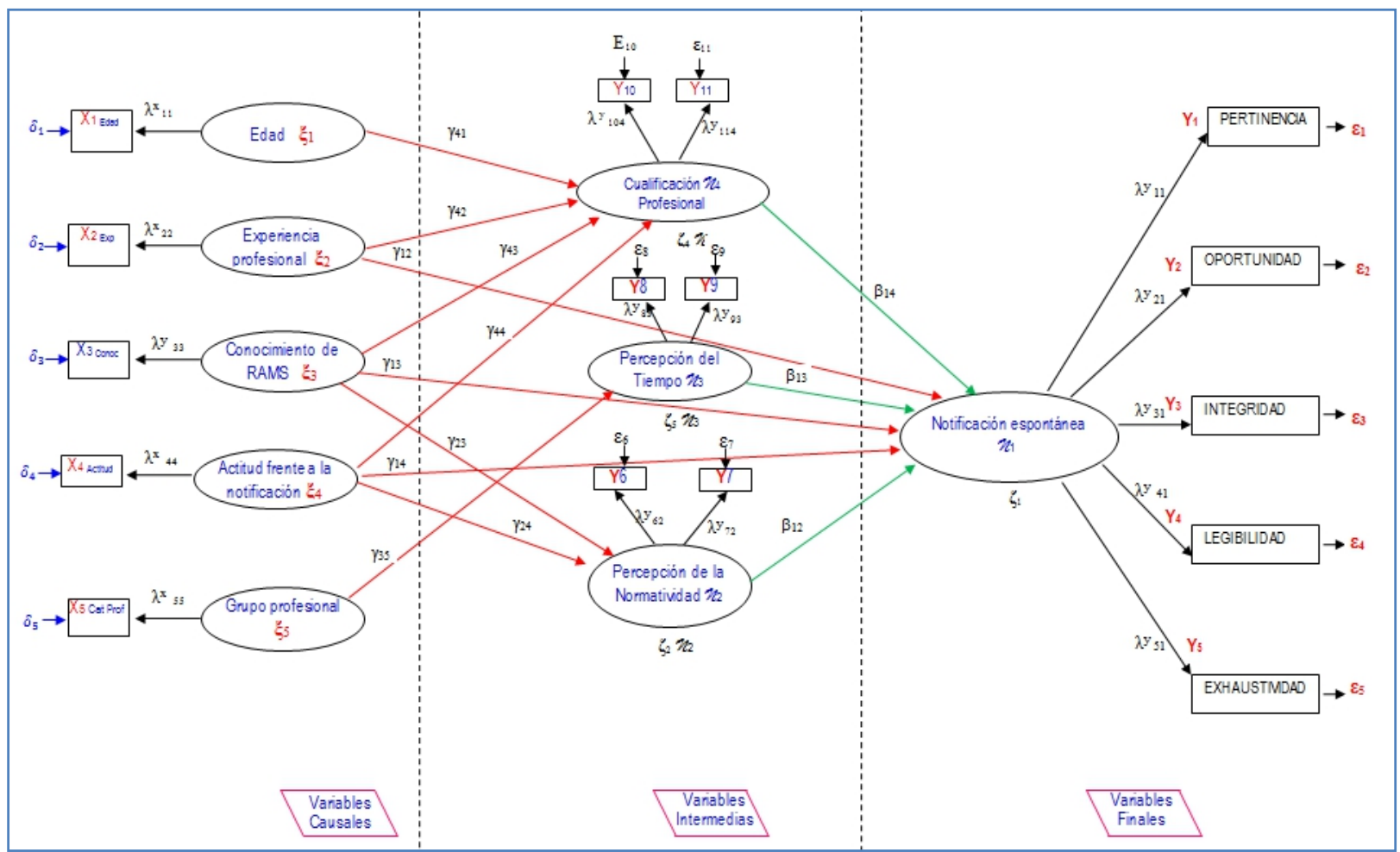

Figura 1. Diagrama de paso del modelo estructural inicial.

Fuente: Elaboración propia.

Posteriormente, se evalúo el ajuste del modelo teórico, observándose que no existe variabilidad en las variables pertinencia ( $\mathrm{Y} 1$ ) y legibilidad (Y4), por lo que

se optó eliminarlas. Las modificaciones se presentan en la figura 2.

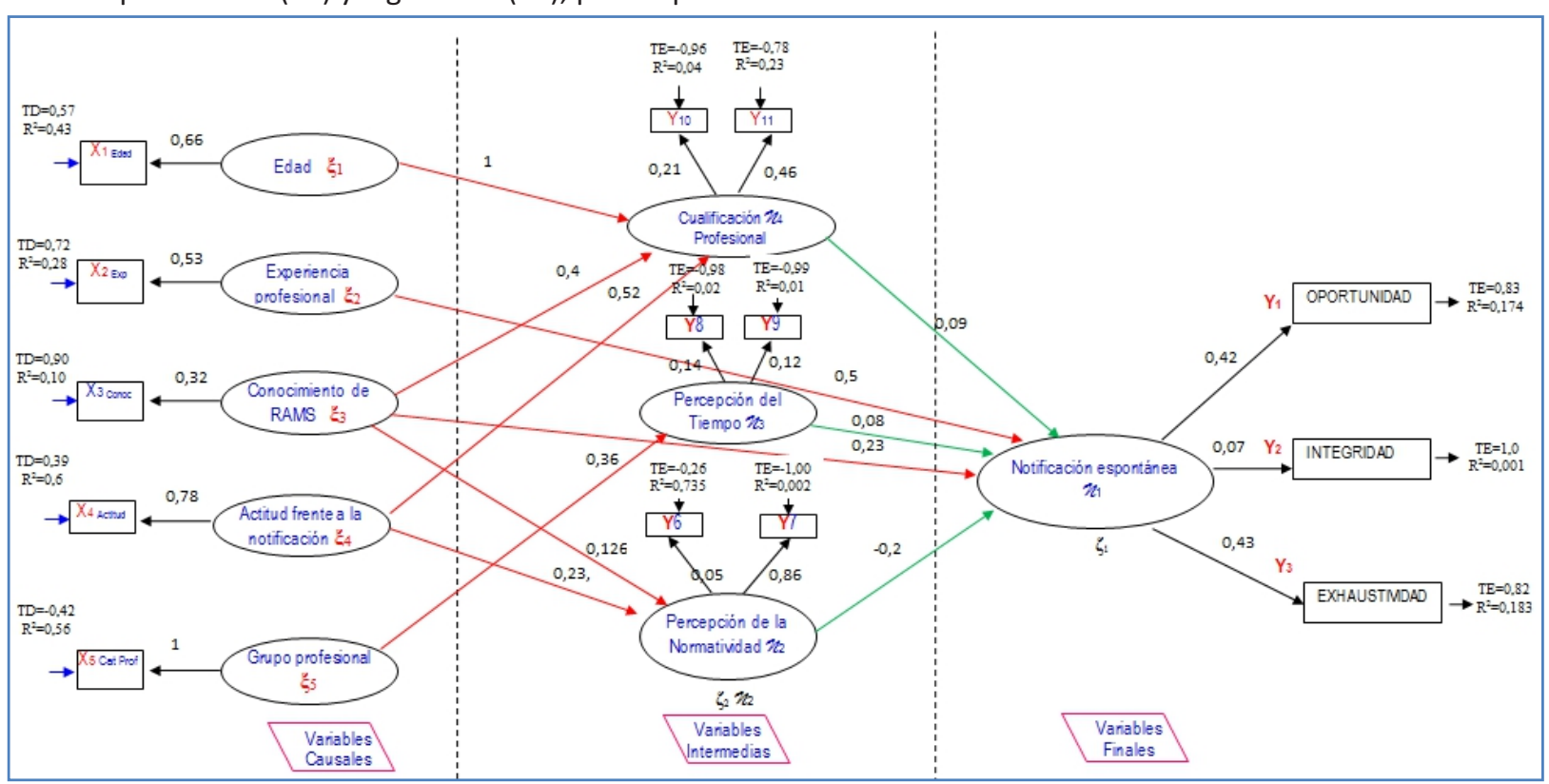

Figura 2. Reespecificación del modelo inicial.

Fuente: Elaboración propia. 
Los índices de bondad de ajuste se encontraron dentro de los límites aceptables. El valor crítico de la prueba (P_value $=0,20387$ ) resultó ser mayor que 0,05 . El índice de ajuste RMSEA $=0,51$ estuvo por debajo del límite de aceptación del 0,08 y el valor de GFI. Seguidamente, se realizó una segunda prueba de ajuste del modelo estableciéndose el modelo final, el cual se presenta a seguir.

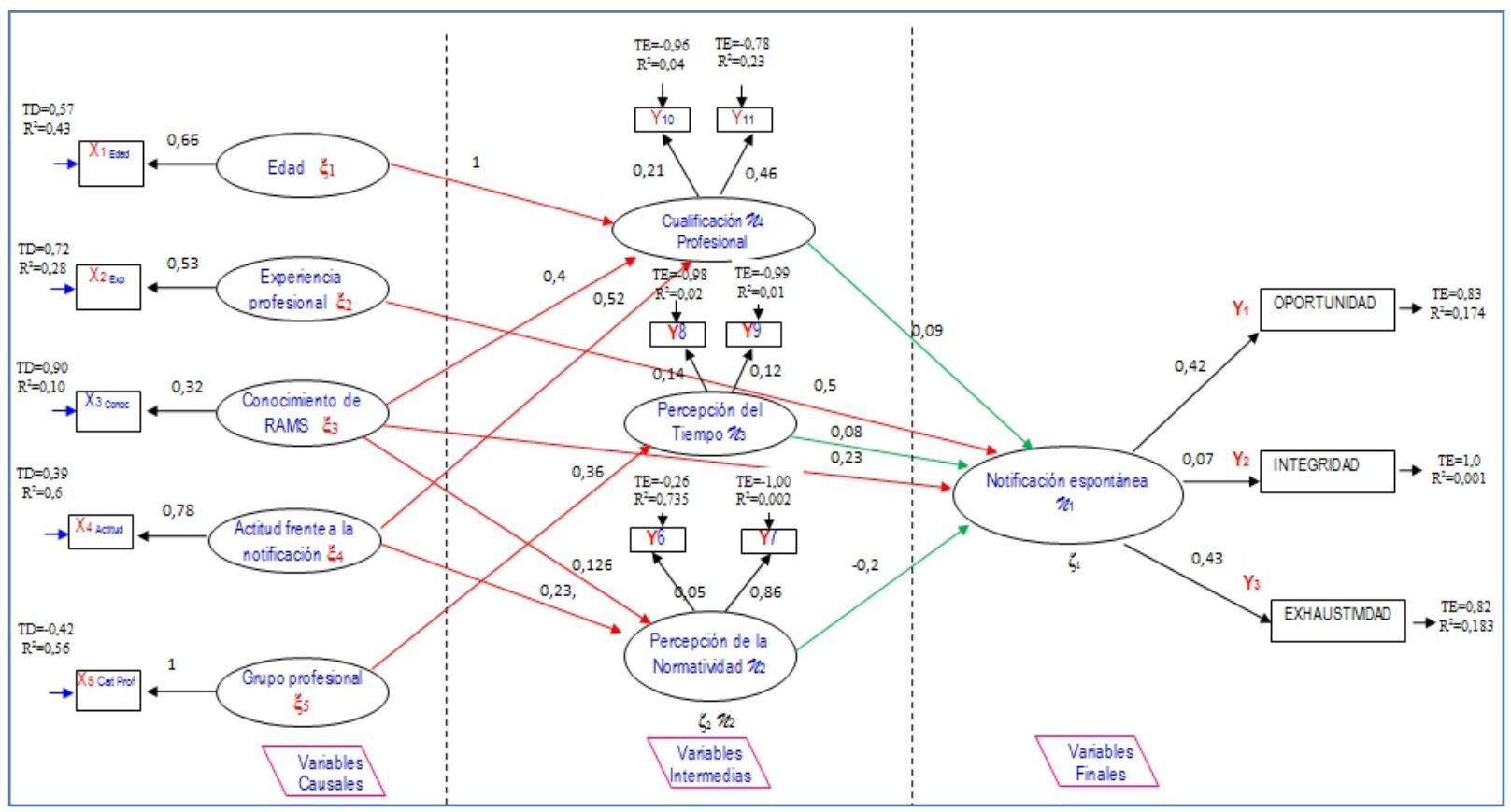

Figura 3. Modelo final.

Fuente: Elaboración propia.

En referencia a la notificación espontánea, se determinó que existen dos indicadores: oportunidad y exhaustividad, cuyos pesos estuvieron en torno a 0,50. Tales valores fueron considerados como buenos indicadores del constructo.

En las relaciones de los constructos exógenos, edad, experiencia profesional, conocimientos de RAMS, actitud frente a la notificación y grupo profesional, los valores resultaron ser superiores a 0,50. Tales indicadores se consideraron como aceptables e incidieron positivamente en el buen ajuste del modelo. En la variable cualificación profesional, existió mayor incidencia de la edad ( $=1$ ) y el conocimiento en RAMS $(=0,52)$; mientras que en relación a la percepción de la normatividad, la variable influyente fue la actitud frente a la notificación $(=0,23)$, versus la variable conocimiento del $\operatorname{RAMs}(=0,126)$.

En el modelo hubo una mayor incidencia de las variables causales que de las variables intermedias sobre la variable notificación espontánea, como el peso del parámetro de la experiencia profesional que incide con un valor de $=0,5$, seguida por el conocimiento de $R A M S$ con un $=0,23$. Por otro lado, las variables intermedias -cualificación profesional y percepción del tiempo- inciden mínimamente con parámetros de efecto directo de $=0,09 \mathrm{y}=0,08$. En cambio, la percepción de la normatividad no contribuye directamente en la notificación espontánea $(=-0,2)$.

\section{DISCUSIÓN}

Las medidas de ajuste global evidenciaron que el modelo final era una representación adecuada del conjunto de relaciones causales entre las variables exógenas y latentes estudiadas. En consecuencia, dado que las medidas de ajuste determinaron el grado en que el modelo (modelo estructural y modelo de medida) predijo globalmente la matriz de datos inicial, se tiene que el modelo había sido adecuado exitosamente.

Se destacó los valores de los parámetros obtenidos en la validación de los modelos de medida de los constructos exógenos y endógenos en el análisis factorial confirmatoria (relación entre variables observadas con las variables latentes), los cuales 
Los índices de bondad de ajuste se encontraron dentro de los límites aceptables. El valor crítico de la prueba (P_value $=0,20387$ ) resultó ser mayor que 0,05 . El índice de ajuste RMSEA $=0,51$ estuvo por debajo del límite de aceptación del 0,08 y el valor de GFI. Seguidamente, se realizó una segunda prueba de ajuste del modelo estableciéndose el modelo final, el cual se presenta a seguir.

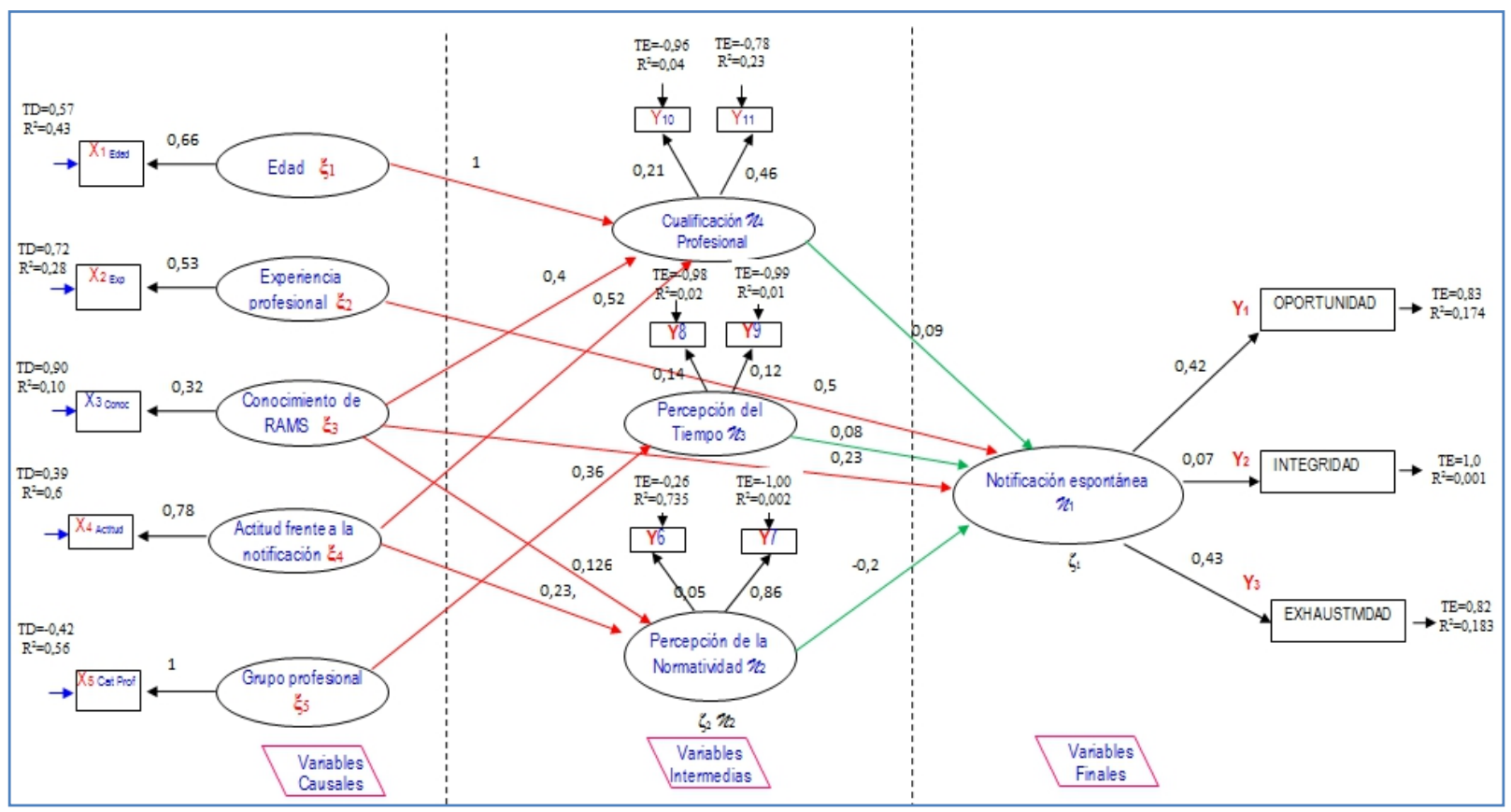

Figura 3. Modelo final.

Fuente: Elaboración propia.

En referencia a la notificación espontánea, se determinó que existen dos indicadores: oportunidad y exhaustividad, cuyos pesos estuvieron en torno a 0,50. Tales valores fueron considerados como buenos indicadores del constructo.

En las relaciones de los constructos exógenos, edad, experiencia profesional, conocimientos de RAMS, actitud frente a la notificación y grupo profesional, los valores resultaron ser superiores a 0,50. Tales indicadores se consideraron como aceptables e incidieron positivamente en el buen ajuste del modelo. En la variable cualificación profesional, existió mayor incidencia de la edad ( $=1$ ) y el conocimiento en RAMS $(=0,52)$; mientras que en relación a la percepción de la normatividad, la variable influyente fue la actitud frente a la notificación $(=0,23)$, versus la variable conocimiento del $\operatorname{RAMs}(=0,126)$.

En el modelo hubo una mayor incidencia de las variables causales que de las variables intermedias sobre la variable notificación espontánea, como el peso del parámetro de la experiencia profesional que incide con un valor de $=0,5$, seguida por el conocimiento de $R A M S$ con un $=0,23$. Por otro lado, las variables intermedias -cualificación profesional y percepción del tiempo- inciden mínimamente con parámetros de efecto directo de $=0,09 \mathrm{y}=0,08$. En cambio, la percepción de la normatividad no contribuye directamente en la notificación espontánea $(=-0,2)$.

\section{DISCUSIÓN}

Las medidas de ajuste global evidenciaron que el modelo final era una representación adecuada del conjunto de relaciones causales entre las variables exógenas y latentes estudiadas. En consecuencia, dado que las medidas de ajuste determinaron el grado en que el modelo (modelo estructural y modelo de medida) predijo globalmente la matriz de datos inicial, se tiene que el modelo había sido adecuado exitosamente.

Se destacó los valores de los parámetros obtenidos en la validación de los modelos de medida de los constructos exógenos y endógenos en el análisis factorial confirmatoria (relación entre variables observadas con las variables latentes), los cuales 
confirmaron la hipótesis de que los indicadores elegidos son una medida adecuada para los constructos.

Respecto a las variables exógenas en el modelo se observaron ocho constructos, tales como la edad, la experiencia profesional, los conocimientos sobre RAMS, la actitud frente a la notificación, el grupo profesional al que pertenece, la cualificación profesional, la percepción del tiempo que emplea para cumplir con la notificación de RAMS y la percepción acerca de la normatividad vigente sobre la materia, los cuales influyeron hipotéticamente de forma directa o indirecta sobre la notificación espontánea de RAMs.

La variable latente final, notificación espontánea, se manifestó a través de tres indicadores: oportunidad, integridad y exhaustividad de la notificación, en los cuales se han hallado pesos altos (valores de $\lambda$ superiores a 0,4$)$. Esto indica que las variables contribuyeron a la definición del constructo notificación espontánea.

En cuanto a las variables intermedias, se tiene la cualificación profesional, la percepción del tiempo que emplea para cumplir con la notificación de RAMS y la percepción acerca de la normatividad vigente sobre la materia.

En lo que respecta a la percepción del tiempo que emplea para cumplir con la notificación de RAMs, el peso encontrado fue de 0,14 (Y8) y 0,12 (Y9), en ambos casos resulta bajo.

En lo concerniente a la variable intermedia percepción sobre la normatividad vigente de la materia, el peso fue de 0,05 (Y6) y de 0,86 (Y7), lo que denota aceptable el valor para Y7 como un buen indicador del constructo.

Asimismo, refiriéndonos a los constructos exógenos o variables causales, en el modelo tenemos cinco: edad; experiencia profesional, conocimiento de RAMS, actitud frente a la notificación y grupo profesional al que pertenece, los que en todos los casos tienen un solo indicador.

Para el primero, edad, se encontró un peso de $\lambda 0,66$, que también resulta un buen indicador; para la variable causal, experiencia profesional, se encontró un peso de $\lambda$ 0,53, también aceptable. En cuanto a los conocimientos que posee el profesional sobre RAMs, el peso hallado fue de $\lambda 0,32$, el que resulta aceptable pero bajo como indicador del constructo. Respecto a la actitud hacia la notificación espontánea de RAMs, el peso obtenido fue de $\lambda 0,78$, por lo que resulta un buen indicador del constructo. Por último, el grupo profesional al cual pertenece tuvo un peso de $\lambda 1$, lo que permite considerarlo también como buen indicador del constructo.

En cuanto a las relaciones entre variables exógenas y la variable endógena, cabe precisar que en el modelo teórico se hipotetizaba una relación directa de la experiencia profesional (y 0,5$)$, conocimiento sobre farmacovigilancia y RAMs $(y, 0,23)$ con la variable final endógena notificación espontánea, la cual se confirma con los parámetros hallados de los valores. Contrariamente, la relación directa hipotética planteado en el modelo de la variable causal actitud frente a la farmacovigilancia y la notificación espontánea con la variable notificación espontánea no se confirmó, sino más bien, se comprobó que la relación entre ambos constructos es indirecta a través de las variables cualificación profesional $(\mathrm{y}=0,52)$ y percepción de la normatividad $(0,23)$.

En cuanto a la variable experiencia profesional, en el modelo teórico inicial, se afirmó que existía una relación directa con la notificación espontánea e indirecta, a través de la variable intermedia cualificación profesional. Sin embargo, se encontró solo una relación directa con la notificación espontánea $(\mathrm{y}=0,50)$, no habiéndose confirmado la relación indirecta.

Respecto a la variable causal conocimiento de farmacovigilancia y RAMS, se hipotetizó una relación directa con la variable final notificación espontánea y una relación indirecta a través de las variables intermedias cualificación y percepción de la normatividad vigente sobre farmacovigilancia y RAMs, confirmándose la relación directa $(0,23)$ y la relación indirecta a través de la variable cualificación profesional $(0,40)$.

En lo tocante a la variable causal actitud frente a la farmacovigilancia y a la notificación de RAMS; se afirmó, en el modelo teórico inicial, que existía una relación directa con la variable final o endógena notificación espontánea e indirecta a través de la cualificación profesional y percepción de la normatividad. Al respecto, se confirmó que la variable causal en mención solo ejerce un efecto indirecto sobre la variable endógena a través de la cualificación profesional y percepción de la normatividad, no 
confirmándose la hipótesis de una relación directa con la variable endógena.

En lo que atañe a la variable causal constructo grupo profesional al que pertenece, se planteó una relación indirecta en el modelo teórico a través de la percepción que tienen los profesionales en cuanto al tiempo que disponen para notificar las RAMS, la cual se confirmó tras la validación del modelo con un valor del parámetro de $\mathrm{y}=0,08$.

En cuanto a las relaciones establecidas en el modelo teórico de las variables intermedias con la variable final notificación espontánea, se confirmaron las hipótesis de que la cualificación profesional y la percepción del tiempo disponible del profesional para cumplir con la notificación espontánea influye directamente sobre la variable endógena notificación espontánea de RAMS, encontrándose efectos significativos en ambos casos ( $\beta=0,09$ para cualificación profesional y $\beta=0,08$ para percepción del tiempo). Se encontró también un efecto indirecto e inverso con la variable percepción de la normatividad $(\beta=-0,2)$.

\section{REFERENCIAS BIBLIOGRÁFICAS}

1. Organización mundial de la salud (OMS). La farmacovigilancia: garantía de seguridad en el uso de los medicamentos Perspectivas políticas de la OMS sobre medicamentos. GinebraSuiza,2004

2. WHO - UMC. Safety Monitoring of Medicinal Product: Guidelines for Setting Up and Running a Pharmacovigilance Centre the Uppsala,Sweden,2000.

3. Moscoso-Veloza, Sonia M.; Ramírez-Cubillos, Gloria F.; LópezGutiérrez, José J. y Gerena-Useche, Bárbara E. Reacciones adversas a medicamentos en el Hospital de Suba de Bogotá. Rev. Salud pública [online]. 2006, vol.8,

4. The Journal of the American Medical Association (JAMA) Incidence of Adverse Drug Reactions in Hospitalized PatientsA Meta-analysis of Prospective Studies Jason Lazarou, MSc; Bruce H. Pomeranz, MD, PhD; Paul N. Corey, PhD JAMA 1998; 279 (15):1200-1205. doi:10.1001/jama.279.15.1200.

5. Peña Espíritu, Nancy Pilar y Echevarría Osorio, Violeta Noemí. Incidencia de reacciones adversas en pacientes hospitalizados del servicio de emergencia del Hospital Nacional Guillermo Almenara Irigoyen Essalud: enero-marzo 2003. Tesis San Marcos Lima, 2004.

6. Espíritu, Nora; Lavado, Gliceria; Pantoja, Lilian; Lam, Carmen; Barrientos, Mónica; Centeno, Rigoberto. Seguridad del paciente Notificación de eventos adversos en un hospital nacional en Lima. Revista de Calidad Asistencial Volumen 22, Issue 6, November 2007, Pages 335-341

7. Rego Hernández, José de Jesús; Leyva de la Torre, Christian y Pérez Sánchez, Magalys. Pesquisaje activo de sospechas de reacciones adversas a medicamentos en el Hospital "Dr.
Salvador Allende: Primer semestre 2006. Revista Cubana de Farmacia, 41(3), 0-0. VOL 41, año 2007.

8. Ministerio de Salud de la Ciudad Autónoma de Buenos Aires. Manual de Farmacovigilancia Hospitalaria Red de Farmacovigilancia Hospitalaria Coordinación de Redes GCBA www.aafhospitalaria.org.ar/.../aafh Manual de Farmacovigiliancia Hosp.

9. Moscoso Veloza, Sonia M; Ramírez Cubillos, Gloria F; López Gutiérrez José J; Gerena Useche Bárbara E. Reacciones Adversas a Medicamentos en el Hospital de Suba de Bogotá. Rev. Salud pública 2006 July, 8(2): 209-217. from: http://www.scielo.org.co/scielo.php?script=sci arttext\&pid=S0124-00642006000200009\&Ing=en.

10. Torelló Iserte J, Castillo Ferrando JR, Laínez MM, García Morillas M, Arias González A. Reacciones adversas a medicamentos notificadas por los médicos de atención primaria de Andalucía: análisis de la infra notificación Atención Primaria (Aten Primaria) 1994 Volumen 13 página(s) 307-11

11. Organización Mundial de la Salud (OMS). Vigilancia de la seguridad de los medicamentos, Guía para la instalación y puesta en funcionamiento de un Centro de Farmacovigilancia. Upsala 2001

12. Bisquerra, R. Introducción conceptual al análisis multivariante. Barcelona. PPU.1989

13. Bentler, P.M. Multivariate analysis with latent variables: Causal modeling. Annual Review of Psychology.1980

14. Riba, M.J. Una panorámica de las técnicas estadísticas multivariantes. En Documentos del Laboratorio de Psicología Matemática. Barcelona: Universidad Autónoma.1987

Fecha de recepción: 12 de junio de 2018

Fecha de aceptación: 10 de noviembre de 2018
Juan José Changllio Roas

E-mail: juanchangllio@yahoo.es 